Biochimica et Biophysica Acta, 592 (1980) 169-184

(C) Elsevier/North-Holland Biomedical Press

BBA 47890

\title{
THE KINETICS OF WATER EXCHANGE ACROSS THE CHLOROPLAST MEMBRANE
}

\author{
R.R. SHARP a and C.F. YOCUM ${ }^{b}$ \\ a Department of Chemistry, University of Michigan, Ann Arbor, MI 48109 and b Division \\ of Biological Sciences and Department of Chemistry, University of Michigan, \\ Ann Arbor, MI 48109 (U.S.A.)
}

(Received January 11th, 1980)

Key words: Chloroplast membrane; Thylakoid volume; Water exchange kinetics; NMR

\section{Summary}

The kinetics of water exchange across the membrane of class II chloroplasts has been studied by two NMR methods. Both methods utilize Dy $(\mathrm{en})^{3+}(\mathrm{en}=$ ethylenediamine) to induce a transmembranal chemical shift the order of 40 $\mathrm{Hz}$ in the water proton resonance. The shift reagent is impermeant to the chloroplast membrane, inert as a redox reagent, soluble at millimolar concentrations at neutral $\mathrm{pH}$, and associated with a large, virtually temperature independent molar shift $(0.10-0.12 \mathrm{ppm} / \mathrm{mM})$. Water exchange across the membrane is monitored by two independent experiments. In the first, chemical exchange causes line broadening in the water proton resonance in the highresolution spectrum. Measurement of the incremental linewidth as a function of transmembranal chemical shift determines the exchange kinetics as well as the fractions of water protons in internal and external media. In the second experiment, chemical exchange causes the transverse relaxation time, as measured by the Carr-Purcell-Gill-Meiboom technique, to be dependent on the $180^{\circ}$ pulse spacing. The two experiments, while independent of each other, depend on the same set of theoretical parameters. These parameters are overdetermined by simultaneous analysis of both experiments. The mean lifetime of a water proton in the inner thylakoid space is found to be $1.1 \pm 0.08 \mathrm{~ms}$ at $25^{\circ} \mathrm{C}$ and $2.75 \pm 0.4 \mathrm{~ms}$ at $3^{\circ} \mathrm{C}$ in $\mathrm{NH}_{2} \mathrm{OH} /$ EDTA-treated chloroplasts. Values derived from dark-adapted chloroplasts that are active with respect to oxygen evolution are $1.1 \pm 0.3 \mathrm{~ms}\left(25^{\circ} \mathrm{C}\right)$ and $1.75 \pm 0.4 \mathrm{~ms}\left(3^{\circ} \mathrm{C}\right)$. The internal thylakoid volume is also determined in principle by the data, but uncertainties in the membrane volume and the transmembranal chemical shift severely limits the accuracy of this measurement. 


\section{Introduction}

It has recently been shown that nuclear magnetic relaxation provides a spectroscopic probe of paramagnetic metal sites in intact thylakoid stacks of broken chloroplasts [1-4]. Both the spin-lattice and spin-spin relaxation times, $T_{1}$ and $T_{2}$, of water protons exhibit interesting variations with magnetic field strength that are indicative of bound $\mathrm{Mn}^{2+}$. Variations in $T_{2}$ are reportedly produced by flash sequences [2], and variations in both $T_{1}$ and $T_{2}$ result from treatments by redox reagents $[1,3,4]$.

An important parameter in the interpretation of magnetic relaxation experiments is the rate of exchange of water protons across the thylakoid membrane. Roughly speaking, water protons sample the magnetic environments of four sites: (1) the inner thylakoid space, (2) the external medium, (3) sites coordinated to bound manganese or other paramagnetic ions, and (4) labile $\mathrm{NH}$ and $\mathrm{OH}$ functional groups that exchange rapidly with solvent protons. When exchange among these sites is very rapid on the NMR timescale, the transverse relaxation is described by a single exponential, the decay rate of which is a weighted average of rates in the different sites. Since water protons in the external medium contribute most of the intensity of the water resonance, the fast exchange condition is necessary for the study of paramagnetic ions bound to the internal membrane.

From considerations of the conditions necessary for monophasic decay, Wydrzynski et al. [4] have inferred limits of $1 \mathrm{~ms}<\tau_{\mathrm{B}}<20 \mathrm{~ms}$ for the mean lifetime of a water proton in the inner thylakoid space. We have also conducted $T_{1}$ and $T_{2}$ measurements in chloroplast suspensions (to be reported elsewhere) and confirm, in dark-adapted chloroplasts, their report of monophasic decay. In several cases $T_{2}$ was not in the exchange-narrowed limit however and showed variations characteristic of transmembranal chemical exchange between chemically shifted media. These chemical exchange effects on $T_{2}$ are magnetic field-dependent, and are qualitatively similar to effects produced by bound paramagnetic ions. In order to facilitate the interpretation of the proton relaxation data, we have measured the transmembranal exchange rate in native and in $\mathrm{NH}_{2} \mathrm{OH} / \mathrm{EDTA}$-treated chloroplasts. These measurements also determins, in principle, the internal thylakoid volume, although in practice the derived volumes appear to have only semiquantitative significance.

Two independent methods of studying transmembranal water exchange have been employed. Both utilize an impermeant shift reagent, Dy $(\mathrm{en})^{3+}(\mathrm{en}=$ ethylenediamine), to induce a chemical shift across the thylakoid membrane. The observed water resonance remains a simple Lorenzian throughout the range of concentrations employed $(0.5-5.0 \mathrm{mM})$, but large effects due to chemical exchange are seen in both the linewidth and the Carr-Purcell decay constant $\left(T_{2}\right)$. These contributions are readily separated by varying the extemal chemical shift (i.e., the Dy(en $)^{3+}$ concentration) or the Carr-Purcell pulse spacing, $t_{\mathrm{cp}}$. Theoretical analysis of the linewidths and $T_{2}$ can then be made to determine (a) the mean lifetimes, $\tau_{\mathrm{A}}$ and $\tau_{\mathrm{B}}$, in the external and internal water pools, and (b) the volume fractions, $P_{\mathrm{A}}$ and $P_{\mathrm{B}}$, of these pools.

Different NMR methods have been applied previously to the study of water exchange in erythrocytes [5-7]. The present method is advantageous in that it 
permits a wider range, especially on the rapid end, of chemical exchange rates that can be studied. Water exchange on the millisecond or submillisecond timescale (which is characteristic of subcellular organelles) is readily monitored by the present technique, while this timescale is accessible only with considerable experimental difficulty, if at all, using previous methods. Another great advantage is that two independent experiments are analyzed using a single set of exchange parameters. Analysis of both experiments simultaneously overdetermines the theoretical parameters and places a stringent criterion of selfconsistency on the overall analysis.

\section{Experimental}

Broken chloroplasts were prepared according to the procedure of Guikema and Yocum [8]. A portion of this material was subsequently extracted with $5 \mathrm{mM} \mathrm{NH} \mathrm{N}_{2} \mathrm{OH}$ plus $1 \mathrm{mM}$ EDTA in the light according to the procedure of Horton and Croze [9]. The buffer contained $0.400 \mathrm{M}$ sucrose, $15 \mathrm{mM} \mathrm{NaCl}$, $0.2 \%$ bovine serum albumin, and $20 \mathrm{mM}$ Tricine $(\mathrm{pH} 8)$. Chloroplasts were suspended in the buffer at concentrations of $2.50 \mathrm{mg} \mathrm{Chl} / \mathrm{ml}$ (native chloroplasts) and $2.190 \mathrm{mg} \mathrm{Chl} / \mathrm{ml}\left(\mathrm{NH}_{2} \mathrm{OH} /\right.$ EDTA extracted chloroplasts). Preparations were divided into aliquots suitable for NMR studies and stored at $-80^{\circ} \mathrm{C}$ until use. Unextracted freeze-thawed chloroplasts retain essentially full activity with respect to oxygen evolution and ATP synthesis. Once thawed, aliquots were discarded after $25 \mathrm{~min}$ of use at $25^{\circ} \mathrm{C}$ or three hours of use at $3^{\circ} \mathrm{C}$. Darkadapted chloroplasts were used in all experiments.

Linewidth measurements on the water resonance were obtained using a high-resolution Fourier transform NMR spectrometer, JEOL JNM/PFT-100, operating at $100 \mathrm{MHz}(23.6 \mathrm{~T})$. Samples consisted of $500 \mu \mathrm{l}$ of chloroplast suspension and $50 \mu \mathrm{l}$ of ${ }^{2} \mathrm{H}_{2} \mathrm{O}$, to which $5-\mu \mathrm{l}$ aliquots of a $50 \mathrm{mM} \mathrm{Dy}(\mathrm{en}) \mathrm{Cl}_{3}$ solution were added. The field was locked on ${ }^{2} \mathrm{H}_{2} \mathrm{O}$. One to ten transients were collected with a delay of 75 seconds, which is greater than $5 T_{1}$ in all samples. Temperature at the sample was controlled to within $\pm 1^{\circ} \mathrm{C}$ using the JEOL temperature controller and measured using a thermocouple in a dummy sample tube. Linewidths at half height were measured directly using the EC-100 data system and have a typical accuracy of $\pm 3 \%$.

$T_{2}$ was measured at $60.0 \mathrm{MHz}$ using a Bruker B/KR-322s spectrometer and a Varian electromagnet. The field was locked on an external ${ }^{7} \mathrm{Li}$ resonance and stabilized with a flux stabilizer. Data from the Carr-Purcell-Gill-Meiboom sequence were sampled and signal averaged (Fabri-Tek Model 1064). In order to circumvent systematic phase shifts that may occur in the Carr-Purcell-GillMeiboom sequence [10], a special detector was used. In this detector the radiofrequency signal is phase-detected not with respect to the carrier, but with respect to a reference radiofrequency source set $50 \mathrm{kHz}$ off-resonance. An active fullwave rectifier followed by an 8-pole Butterworth filter set at 25 $\mathrm{kHz}$ is then used as an envelope detector. The resulting signal is proportional to the modulus of the transverse magnetization; it is equivalent to that from a diode detector but is highly linear down to millivolt radiofrequency levels. The absolute accuracy of $T_{2}$ measurements was tested in the following manner. Degassed water $(\mathrm{pH} 3)$ was sealed in a sample tube and $T_{1}$ was measured as a 
function of temperature using the triplet sequence. The data agreed closely with literature values of Simpson and Carr [11]. Meiboom [12] has shown that $T_{1}$ equals $T_{2}$ in water at $\mathrm{pH}$ values below $4 . T_{2}$ was then measured and found to equal $T_{1}$ within typically $1-3 \% . T_{2}$ of the reference water sample was measured frequently to check the experimental accuracy.

The shift reagent was prepared by dissolving $\mathrm{Dy}_{2} \mathrm{O}_{3}$ (Alpha Chemical Co., $99.9 \%)$ in $0.600 \mathrm{M} \mathrm{HCl}$ to give a solution that is $0.200 \mathrm{M}^{\text {in }} \mathrm{Dy}^{3+}$. Ethylenediamine was dissolved to $50 \mathrm{wt} \%$ in water and titrated to $\mathrm{pH} 6.5 \mathrm{with} \mathrm{HCl}$. This solution was diluted with water to give a solution $20 \%$ ethylenediamine by weight. The neutralized ethylenediamine was added in equimolar concentration to the $\mathrm{Dy}^{3+}$ solution, and then titrated with $\mathrm{KOH}$ to the first appearance of precipitate ( $\mathrm{pH}$ 6.25). The resulting solution, which is $50 \mathrm{mM}$ in Dy(en $)^{3+}$, was added in $5 \mu \mathrm{l}$ aliquots to the buffered chloroplast suspension.

The molar chemical shift induced by Dy $(\mathrm{en})^{3+}$ in the water proton resonance of the buffer was measured directly using benzene in a sealed capillary as external reference. Measured values of the dysprosium shift were near 0.11 $\mathrm{ppm} / \mathrm{mM}$ and were stable over a period of at least six weeks. Surprisingly, the molar shift of Dy(en $)^{3+}$ is essentially temperature-independent between $3^{\circ} \mathrm{C}$ and $25^{\circ} \mathrm{C}$. This behavior is not in general expected for either transition metal ions or Lanthanide ions [13-16] and apparently reflects a cancellation of effects, possibly including temperature-dependent shifts in the hydrolysis equilibria.

The permeability of Dy(en $)^{3+}$ toward the chloroplast membrane was tested by monitoring the linewidth in chloroplast suspensions as a function of time after the addition of shift reagent (Fig. 1). It is shown below that this linewidth results from chemical exchange contributions associated with the transfer of water molecules across the thylakoid membrane and between regions of differing chemical shift. Leakage of the shift reagent across the thylakoid membrane

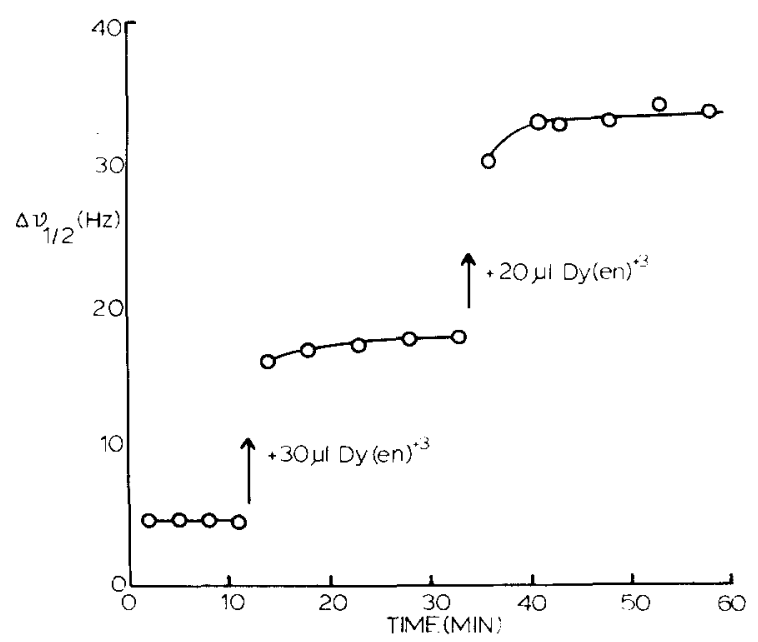

Fig. 1. Time course of the proton linewidth after addition of Dy(en $)^{3+}$. Aliquots of $50 \mathrm{mM} \mathrm{Dy(en)^{3+ }}$ (as $^{3}$ the chloride) were added to an NMR tube containing $500 \mu \mathrm{l}$ of chloroplast suspension $(2.190 \mathrm{mg} / \mathrm{ml}$ of $\mathrm{NH}_{2} \mathrm{OH} /$ EDTA-treated chloroplasts in the buffer) and $50 \mu \mathrm{l}^{2} \mathrm{H}_{2} \mathrm{O}$. Experiments were conducted in the dark at a proton frequency of $100 \mathrm{MHz}, T=25^{\circ} \mathrm{C}$. 
would collapse the chemical shift difference and restore the linewidth to the value prior to addition of the aliquot. The linewidth exhibits a step increase followed by a slower rise during the first three or four minutes after addition of shift reagent. It is then stable over intervals of at least $20 \mathrm{~min}$. The slow portion of the initial rise may reflect hydrolysis equilibria involving $\operatorname{Dy}(e n)^{3+}$, since a similar drift of about $0.15 \mathrm{pH}$ units is also seen in the $\mathrm{pH}$. In subsequent experiments, measurements were taken three minutes after the addition of shift reagent.

\section{Theoretical}

\section{Two-site model of chemical exchange}

Chemical exchange of water protons across the thylakoid membrane in general produces broadening of the proton resonance when the chemical shift of the resonance differs in the internal and external aqueous phases. A simple qualitative picture of the phenomenon is as follows. In the absence of chemical exchange, two resonances are observed at angular frequencies $\omega_{A}$ and $\omega_{B}$, corresponding to protons in the external and internal sites, respectively. When water molecules exchange between the two sites at a rate that is comparable to the chemical shift difference, $\delta \equiv \omega_{\mathrm{A}}-\omega_{\mathrm{B}}$, the resonances broaden and coalesce. At exchange rates very rapid compared to $\delta$, a single exchangenarrowed resonance is observed, the residual linewidth of which is determined by the mean lifetimes, $\tau_{\mathrm{A}}$ and $\tau_{\mathrm{B}}$, and the volume fractions, $P_{\mathrm{A}}$ and $P_{\mathrm{B}}$, of water in the two sites. In the following these situations are referred to as slow exchange (two resolved but broadened resonances), intermediate exchange (the region of coalescence), and fast-exchange (a single exchange-narrowed resonance). Chloroplast suspensions have been studied only in the fast exchange and coalescence regions.

Although a two-site model is a gross simplification of the thylakoid structure, it appears to be appropriate for the interpretation of NMR measurements. In the external medium, the solvent protons exchange rapidly on the NMR timescale with the sites in the hydration sphere of $D y(e n)^{3+}$. Furthermore, diffusive mixing of water occurs rapidly over regions comparable in dimension to the thylakoid stack. This can be shown by calculating the mean-square displacement, $\bar{r}^{2}=6 D t$, of a molecule diffusing in three dimensions during a time interval $t$. If the diffusion coefficient is taken to be the value for pure water [11], $D=2.1 \cdot 10^{-5} \mathrm{~cm}^{2} \cdot \mathrm{s}^{-1}$ the time required for water protons to achieve a root-mean-square displacement comparable to the dimension of a thylakoid $(500 \mathrm{~nm})$ is of order $5 \mu \mathrm{s}$. This is more than two orders of magnitude shorter than the mean lifetime of a water molecule in the inner thylakoid space at $25^{\circ} \mathrm{C}$ (see below), and is about two orders of magnitude faster than the shortest pulse spacings in our experiments. Thus, diffusion effectively averages the magnetic environment of water in the external phase. (This approximation is of marginal validity for particles the size of erythrocytes, however.)

It is not known with certainty whether the inner aqueous space of the thylakoid stack and fretwork forms a single enclosed volume with continuity throughout the aqueous phase, but the extensive electron microscopic studies of Paolillo [17-23] strongly support this conclusion. Certainly the inner 
volume of stroma lamellae is contiguous with that of granal lamellae [20]. Reconstruction of the three-dimensional structure of the lamellae and fretwork using serial sections indicates that a helical structure exists in which the stromal lamellae interconnect sequentially with each granum of the stack. Conversely, each granum interconnects with several stroma lamellae. A continuous enclosed aqueous space between and including separated grana has been demonstrated [20]. As indicated above, diffusion of water is sufficiently fast to assure that the transfer of protons between grana is rapid on the NMR timescale. Thus, the internal medium should very probably be viewed, from the standpoint of proton NMR measurements, as a continuous aqueous space that includes the loculi and fretwork of several interconnected thylakoids. As long as water diffuses rapidly throughout the internal aqueous space, the specific geometry of the chloroplast ultrastructure has no influence on the analysis of the NMR experiment. Only the fraction of water molecules in the internal aqueous space and the mean lifetime of a water proton in this space are relevant parameters. It is possible that the absolute internal volumes of individual chloroplasts may not be uniform throughout the suspension. This kind of structural nonuniformity appears to be quite unimportant, however, since the exchange kinetics depend on the surface to volume ratio of the chloroplast, rather than on the absolute internal volume.

\section{Chemical exchange effect on the high-resolution lineshape}

The effect of chemical exchange on the NMR lineshape can be calculated using the modified Block equations [24], or alternatively, by the density matrix approach of Anderson [25,26]. An analytical solution to the modified Block equation has been given by Rogers and Woodbrey [27] for the general two site case:

$v=\frac{-\gamma H_{1} M_{\mathrm{o}}\left\{P\left(1+\tau R_{2}^{\circ}\right)+Q S\right\}}{Q^{2}+S^{2}}$

where

$P \equiv \tau\left[R_{2 \mathrm{~A}} R_{2 \mathrm{~B}}-\delta^{2}+\left(\frac{\omega-\omega_{0}}{2}\right)^{2}\right]+R_{2}^{\circ}$

$Q \equiv \tau\left[\delta-\left(\frac{\omega-\omega_{0}}{2}\right)\left(P_{\mathrm{A}}-P_{\mathrm{B}}\right)\right]$

$S \equiv\left(\omega-\omega_{0}\right)\left[\left(1+\tau R_{2}^{\circ}\right)+\left(\frac{\tau}{2}\right)\left(R_{2 \mathrm{~B}}-R_{2 \mathrm{~A}}\right)+1 / 2\left(P_{\mathrm{A}}-P_{\mathrm{B}}\right)\right]$.

$v$ is proportional to the signal amplitude in the absorption mode. $R_{2 \mathrm{~A}}$ and $R_{2 \mathrm{~B}}$ are transverse relaxation rates in the $A$ and $B$ sites, $\omega$ is the radiofrequency, $H_{1}$ is the radiofrequency field strength, $\gamma$ is the magnetogyric ratio, $M_{\mathrm{o}}$ is the equilibrium bulk magnetization, $\omega_{0}=1 / 2\left(\omega_{A}+\omega_{B}\right)$, and $\tau^{-1}=\tau_{A}^{-1}+\tau_{B}^{-1}$.

Equation 1 describes the resonance lineshape of the absorption mode. The shape is Lorenzian only in the fast exchange region, in which case the full width at half-height is given by

$\Delta \nu_{1 / 2}=\left(\pi T_{2}\right)^{-1}=\pi^{-1} P_{\mathrm{A}} P_{\mathrm{B}} \delta^{2} \tau$ 
In the present work, the linewidth of the water proton resonance is monitored as a function of increasing chemical shift between internal and external volumes. For this experiment it is convenient to express Eqn. 2 in terms of the following variables: (a) the total added volume of shift reagent, $V_{\mathrm{SR}}$; (b) the internal thylakoid volume, $V_{\mathrm{i}}$; (c) the membrane volume, $V_{\mathrm{m}}$; and (d) the total volume prior to addition of shift reagent, $V_{\mathrm{T}}$. Making the substitutions, $P_{\mathrm{B}}=V_{\mathrm{i}} /\left(V_{\mathrm{T}}-V_{\mathrm{m}}\right), P_{\mathrm{A}}=1-P_{\mathrm{B}}$, and $\tau=P_{\mathrm{A}} \tau_{\mathrm{B}}=P_{\mathrm{B}} \tau_{\mathrm{A}}$, Eqn. 2 can be written

$$
\Delta \nu_{1 / 2}=\frac{\left(2 \pi \sigma M_{\mathrm{SR}}\right)^{2}\left(V_{\mathrm{i}} \tau_{\mathrm{B}}\right) V_{\mathrm{SR}}^{2}}{\pi\left(V_{\mathrm{T}}-V_{\mathrm{m}}+V_{\mathrm{SR}}\right)^{3}}
$$

where $\sigma$ and $M_{\mathrm{SR}}$ are the molar shift $\left(\mathrm{Hz} \cdot \mathrm{M}^{-1}\right)$ and molarity of Dy $(\mathrm{en})^{3+}$ in the aliquots. This result is valid in the fast-exchange region. According to Eqns. 1 and 3 a plot of $\Delta \nu_{1 / 2}$ vs. $V_{\mathrm{SR}}^{2}$ is linear as $V_{\mathrm{SR}} \rightarrow 0$. As $V_{\mathrm{SR}}$ increases, $\Delta \nu_{1 / 2}$ shows a negative deviation from the limiting slope and passes through a maximum in the coalescence region.

Fig. 2 illustrates the theoretical dependence of the linewidth on $\tau_{B}$ (the mean lifetime in the thylakoid space) for volume fractions typical of the chloroplast suspensions we have used (details are given in the figure legend and correspond to an internal volume of $80 \mu \mathrm{l} / \mathrm{mg} \mathrm{Chl}$ and a membrane volume of $133 \mu \mathrm{l} / \mathrm{mg}$ Chl). It is evident that the initial slope and coalescence point are sensitive functions of the exchange parameters. It should be noted that although the resonance is not Lorenzian outside the fast exchange region, $\Delta \nu_{1 / 2}$ is defined as the width at half-height of the lineshape given by Eqn. 1 and is readily determined by computer simulation.

\section{Effect of chemical exchange on the Carr-Purcell train}

The Carr-Purcell train measures the rate of irreversible decay of magnetiza-

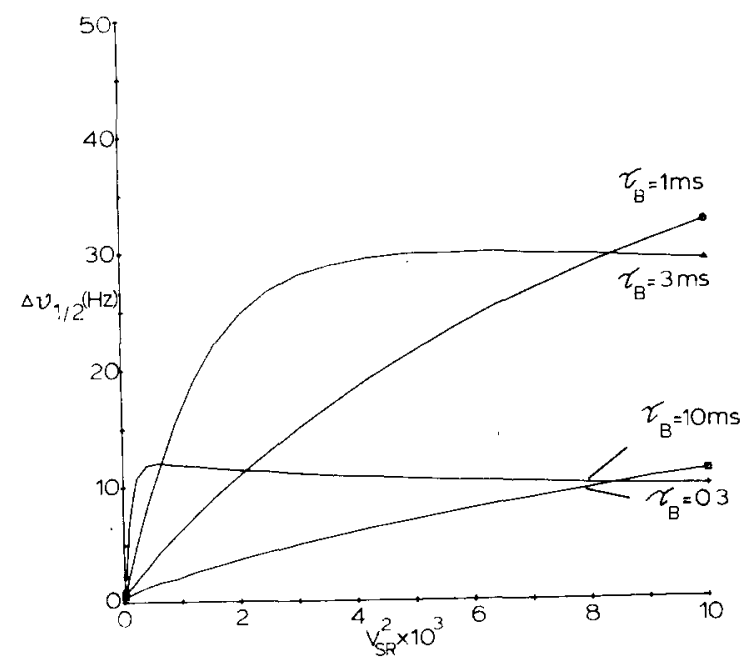

Fig. 2. Theoretical results of a type $I$ experiment for various values of $\tau_{B}$, the mean lifetime of water molecules in the internal site. Assumed parameters are $V_{\mathrm{m}}=133 \mu \mathrm{l}, V_{\mathrm{i}}=80 \mu \mathrm{l}, V_{\mathrm{T}}=550 \mu \mathrm{l} ;$ the molar shift and molarity of the shift reagent solution are $0.10 \mathrm{ppm} / \mathrm{mM}$ and $50 \mathrm{mM}$. 
tion in a plane transverse to the external field (taken to lie along the $z$-direction). The following pulse train is employed: $90(0)-\left(t_{\mathrm{cp}} / 2\right)\left(180(\pi / 2)-t_{\mathrm{cp}}-\right)_{n}$. $90(0)$ is a $90^{\circ}$ pulse with zero radiofrequency phase shift; the $180^{\circ}$ pulses are phase-shifted by $\pi / 2$ (the Gill-Meiboom modification) [28,29]. Irreversible dephasing of proton spins results from three causes: (1) molecular diffusion of the resonant nuclei in an inhomogeneous magnetic field. Diffusion causes the precession frequencies of individual spins to vary in a random manner, thus leading to an irreversible loss of phase coherence; (2) chemical exchange between chemically-shifted sites. Random migration of spins into sites of differing chemical shifts causes a random fluctuation of spin precession frequencies and an irreversible loss of phase coherence; (3) $T_{2}$ processes arising from fluctuating intermolecular magnetic fields (various relaxation mechanisms are described elsewhere) $[29,30]$. The chemical exchange contribution can be studied selectivity by observing the dependence of the relaxation rate, $R_{2} \equiv$ $1 / T_{2}$, on the pulse spacing, $t_{\mathrm{cp}}$.

Use of the Carr-Purcell train in studies of chemical exchange is described by Bloom, Reeves and Wells [31] and by Gutowsky and coworkers [32-34]. Most convenient for the present case is the general two-site analysis of Allerhand and Gutowsky [33], in which the following analytical expression is derived for the Carr-Purcell decay constant, $T_{2}$ :

$T_{2}^{-1}=\left(T_{2}^{0}\right)^{-1}+(2 \tau)^{-1}-t_{\mathrm{c} \mathrm{p}}^{-1} \sinh ^{-1} F$

$F=\left[D_{+} \sinh ^{2}\left(t_{\mathrm{cp}} S_{\mathrm{r}} / 2\right)+D_{-} \sin ^{2}\left(t_{\mathrm{cp}} S_{\mathrm{i}} / 2\right)\right]^{1 / 2} ;$

$2 D_{ \pm}= \pm 1+\left[(1 / \tau)^{2}+(\delta \omega)^{2}\right]\left(S_{\mathrm{r}}^{2}+S_{\mathrm{i}}^{2}\right)^{-1} ;$

$S_{\mathrm{r}}$ and $S_{\mathrm{i}}$ are real and imaginary parts of

$S=\left[\left(\frac{1}{\tau}\right)^{2}-(\delta)^{2}+2 i\left(P_{\mathrm{a}}-P_{\mathrm{b}}\right)(\delta / \tau)\right]^{1 / 2}$.

$T_{2}^{0}$ is the decay constant in the absence of chemical exchange. The chemical exchange contribution, $T_{2}^{-1}-\left(T_{2}^{0}\right)^{-1} \equiv T_{\mathrm{e}}^{-1}$, depends on the four parameters $t_{\mathrm{cp}}, \delta, P_{\mathrm{B}}$ and $\tau$ (where $\tau^{-1}=\tau_{\mathrm{A}}^{-1}+\tau_{\mathrm{B}}^{-1}$ ).

In a typical experiment, $R_{2} \equiv T_{2}^{-1}$ is monitored as a function of $t_{\mathrm{cp}}$ at fixed temperature (constant $\tau$ ), fixed concentration of shift reagent (constant $\delta$ ), and fixed chloroplast concentration (fixed $P_{\mathrm{A}}, P_{\mathrm{B}}$ ). (This type of measurement will be termed experiment II.) Theoretical plots corresponding to volume fractions typical of the chloroplast suspensions we have used are shown in Fig. 3. (The conditions are the same as in Fig. 1.) $R_{2}-R_{2}^{0}$ rises in a sigmoidal fashion with increasing $t_{\mathrm{cp}}$, with the inflection point occurring when $t_{\mathrm{cp}} \cong 2 \tau$. Chemical exchange effects are 'pulsed away' in the rapid pulsing limit, where all curves approach a common limiting value equal to $R_{2}^{0}$. The inflection point of the curve is determined principally by $\tau$; in fact when $R_{2 \mathrm{~A}}=R_{2 \mathrm{~B}}>t_{\mathrm{cp}}$ and $\delta<<(2 \tau)^{-1}$, (conditions that are approximately satisfied in our experiments) Eqn. 4 reduces to

$$
R_{2}-R_{2}^{\circ}=P_{\mathrm{A}} P_{\mathrm{B}} \delta^{2} \tau\left[1-\left(\frac{2 \tau}{t_{\mathrm{cp}}}\right) \tanh \left(\frac{t_{\mathrm{cp}}}{2 \tau}\right)\right]
$$




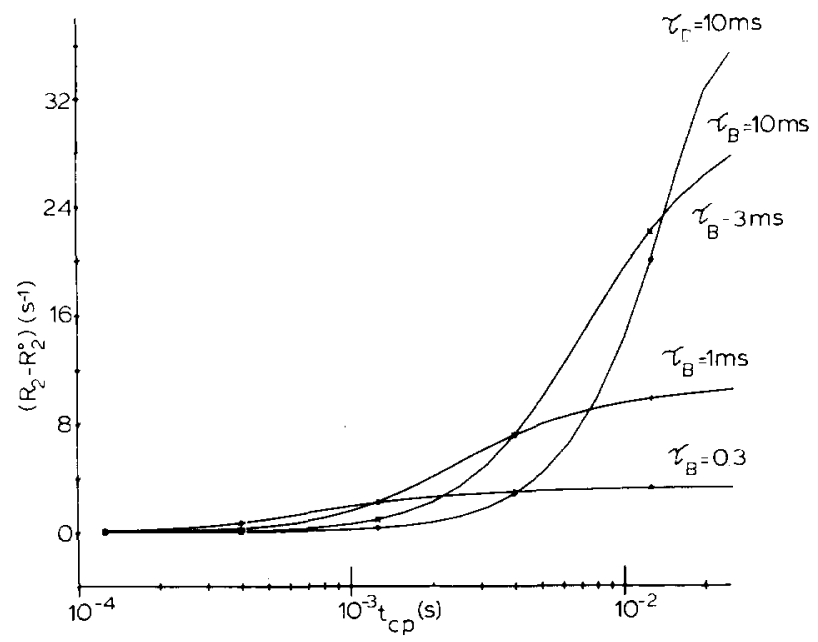

Fig. 3. Theoretical results of a type II experiment for various values of $\tau_{\mathbf{B}}$. Assumed parameters are the same as those in Fig. 2.

In this approximation the inflection point is determined entirely by the exchange rate, and is independent of $\delta, P_{\mathrm{A}}$ and $P_{\mathrm{B}}$.

\section{Results}

\section{$\mathrm{NH}_{2} \mathrm{OH} / \mathrm{EDTA}$-treated chloroplasts}

Chloroplasts were extracted in the light with $5 \mathrm{mM} \mathrm{NH}_{2} \mathrm{OH}, 1 \mathrm{mM}$ EDTA to remove extractable $\mathrm{Mn}^{2+}$, and resuspended in the buffer at a concentration of $2.190 \mathrm{mg}$ chlorophyll $/ \mathrm{ml}$. With no addition of $\mathrm{Dy}(\mathrm{en})^{3+}$, the linewidth of water protons at $100 \mathrm{MHz}$ is $4.0 \pm 0.5 \mathrm{~Hz}$, while that in the buffer is $2.0 \mathrm{~Hz}$. Addition of 5- $\mu \mathrm{l}$ aliquots of $\mathrm{Dy}(\mathrm{en})^{3+}(550 \mu \mathrm{l}$ total volume) produces rapid line broadening in the high resolution spectrum as shown in Fig. 4. Plotted linewidths have been corrected for the effect of $\mathrm{Dy}(\mathrm{en})^{3+}$ on the buffer, a correction that is about $10 \mathrm{~Hz}$ at the maximum concentration of Dy(en $)^{3+}$. According to Eqn. 3, quadratic dependence of $\Delta \nu_{1 / 2}$ on $V_{\mathrm{SR}}$ is expected in the fast-exchange region, and this behavior is in fact observed. At higher concentrations of shift reagent, the linewidth passes through a maximum.

$T_{2}$ as measured by the Carr-Purcell technique showed a dependence on $t_{\mathrm{cp}}$ that is likewise indicative of a chemical exchange contribution (Fig. 5). The two curves in Fig. 5 show the dependence of $R_{2}$ on $t_{\mathrm{cp}}$ when $30 \mu \mathrm{l}$ and $40 \mu \mathrm{l}$ aliquots of Dy(en $)^{3+}$ are added to $500 \mu \mathrm{l}$ of suspension. $T_{2}^{-1}$ increases monotonically with $t_{\mathrm{cp}}$ and exhibits an inflection point at the same value of $t_{\mathrm{cp}}$, approx. $2.5 \mathrm{~ms}$, for both concentrations of shift reagent. Data near $400 \mu \mathrm{s}$ are essentially in the rapid pulse limit, where chemical exchange effects are 'pulsed away'. Pulse spacings longer than $10 \mathrm{~ms}$ were not experimentally feasible since $t_{\mathrm{cp}} \sim T_{2}$ in this region. An approximate value of $\tau, \tau \equiv\left(\tau_{\mathrm{A}}^{-1}+\tau_{\mathrm{B}}^{-1}\right)^{-1}=1.2 \mathrm{~ms}$, can be obtained by inspection from the inflection point of the $t_{\mathrm{cp}}$ plot. Control experiments showed no systematic variation of $T_{2}$ with $t_{\mathrm{cp}}$ in the absence of shift reagent. Measurements on chloroplast suspensions were generally inter- 


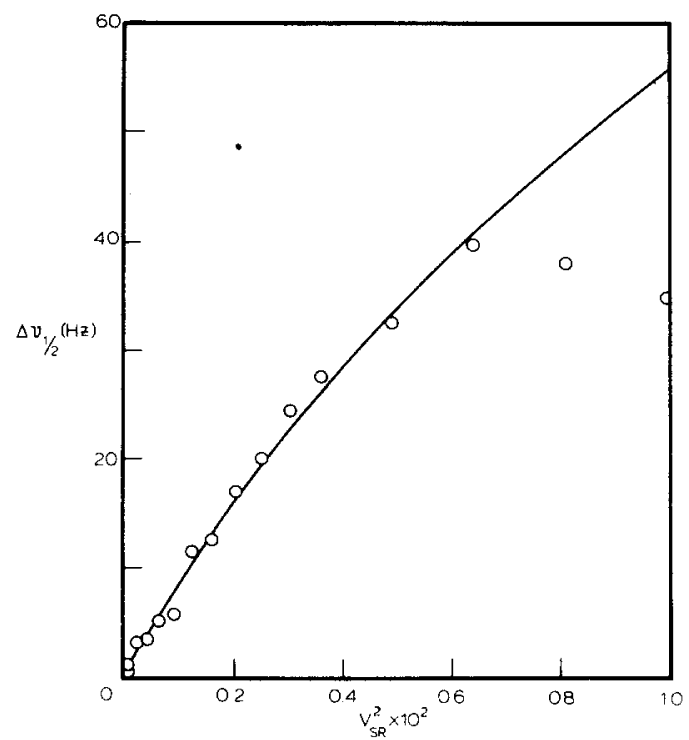

Fig. 4. Dependence of the resonance linewidth of the water protons on the volume squared of added shift reagent $\left(V_{\mathrm{SR}^{2}}{ }^{2}\right) .5-\mu$ l aliquots of the shift reagent $\left(50 \mathrm{mM} \mathrm{Dy}(\mathrm{en}) \mathrm{Cl}_{3}, \mathrm{pH} 6.25\right)$ were added to a solution containing $50 \mu \mathrm{l}{ }^{2} \mathrm{H}_{2} \mathrm{O}$ and $500 \mu \mathrm{l}$ dark-adapted chloroplasts. Chloroplasts $(2.190 \mathrm{mg} / \mathrm{ml})$ were extracted with $5 \mathrm{mM} \mathrm{NH} \mathrm{mH}_{2} \mathrm{OH}$ and washed with EDTA as described in Methods. $T=24.5 \pm 0.5^{\circ} \mathrm{C}$. The solid line is a computer fit using Eqn. 1 of the text. Fitted parameters are given in Table $I$.

spersed with measurements on doped water to exclude the possibility of systematic error in the $T_{2}$ measurement.

The data in Figs. 4 and 5 have been fit to Eqns. 3 and 5 using a nonlinear least-squares fitting program, BMDX-85, obtained from the Statistics Research Laboratory of the University of Michigan. Theoretical curves (shown as the solid lines in Figs. 4-9) were initially generated by iterations on the parameters

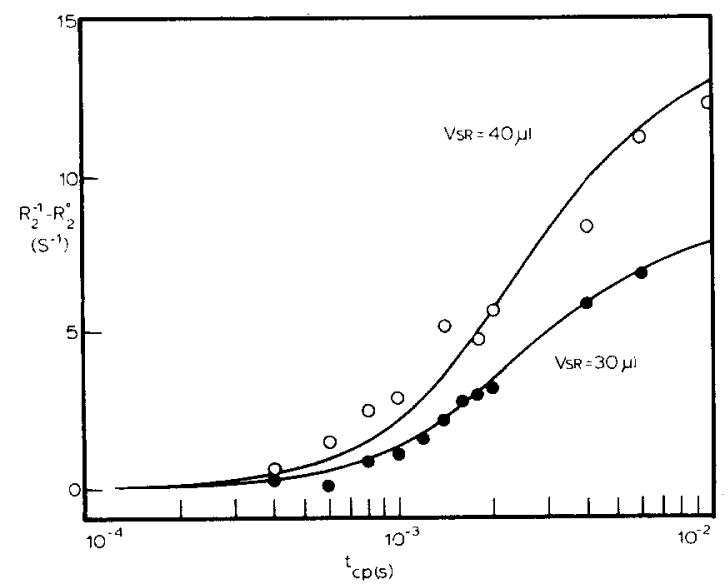

Fig. 5. Dependence of the Carr-Purcell-Gill-Meiboom decay rate on the $180^{\circ}$ pulse spacing. For details of the chloroplast suspension, see Fig. 4. $\mathrm{T}=24.5 \pm 0.5^{\circ} \mathrm{C}$. Proton frequency equals $60.0 \mathrm{MHz}$. Total volume equals $500 \mu \mathrm{l}$ plus the indicated volumes of added shift reagent $\left(V_{\mathrm{SR}}\right)$. In the absence of shift reagent, $T_{2}^{-1}=3.5 \mathrm{~s}^{-1}$ at $25^{\circ} \mathrm{C}$, when $t_{\mathrm{cp}}=1 \mathrm{~ms}$. The solid line is a computer fit using Eqn. 4 of the text. 


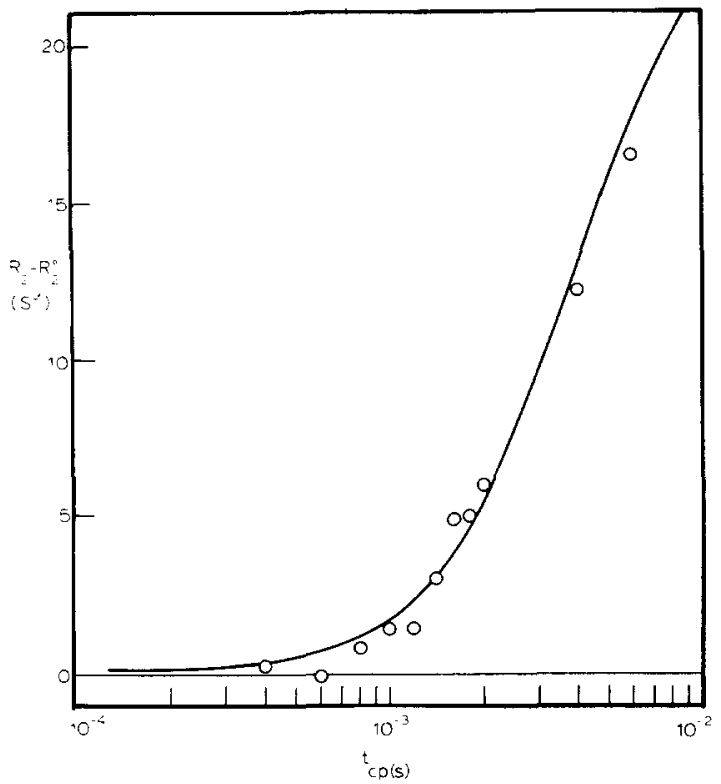

Fig. 6. Temperature dependence of $R_{2}$ in the Carr-Purcell-Gill-Meiboom experiment. Same conditions as for the open circles in Fig. 5. The solid line represents the theoretical fit obtained under assumptions outlined in the text.

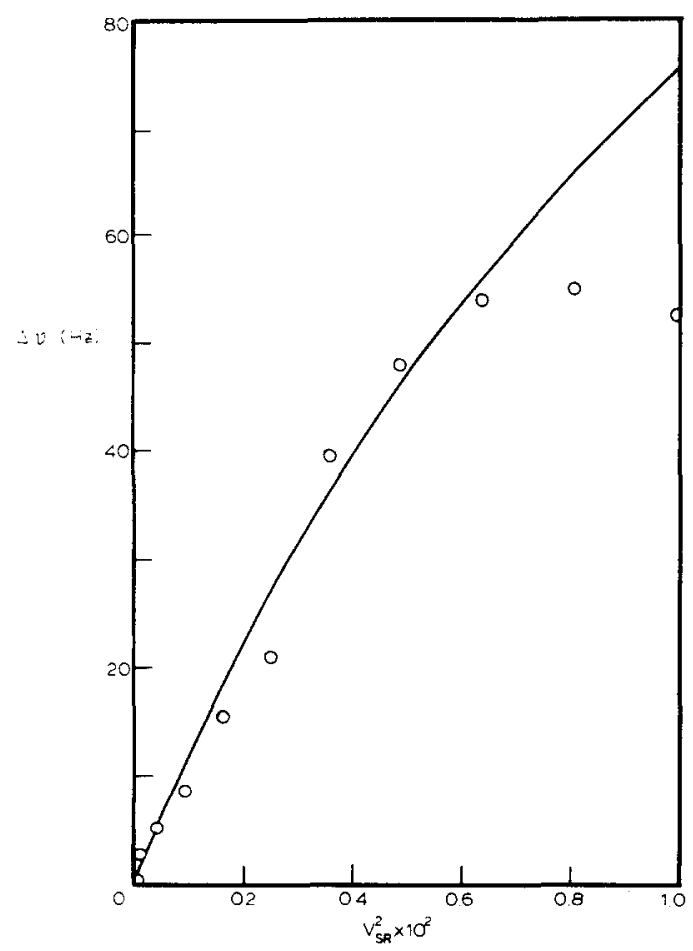

Fig. 7. Results of experiment $I$ of the text for a native chloroplast suspension $T=25^{\circ} \mathrm{C}$, proton frequency is $100 \mathrm{MHz}$. The sample contains $500 \mu \mathrm{l}$ dark-adapted chloroplasts $(2.50 \mathrm{mg} \mathrm{Chl} / \mathrm{ml}), 50 \mu \mathrm{l}{ }^{2} \mathrm{H}_{2} \mathrm{O}$, plus the indicated volume $\left(V_{S R}\right)$ of shift reagent solution. The solid line is the result of a computer fit to Eqn. 1 using the theoretical parameters listed in Table 1. 


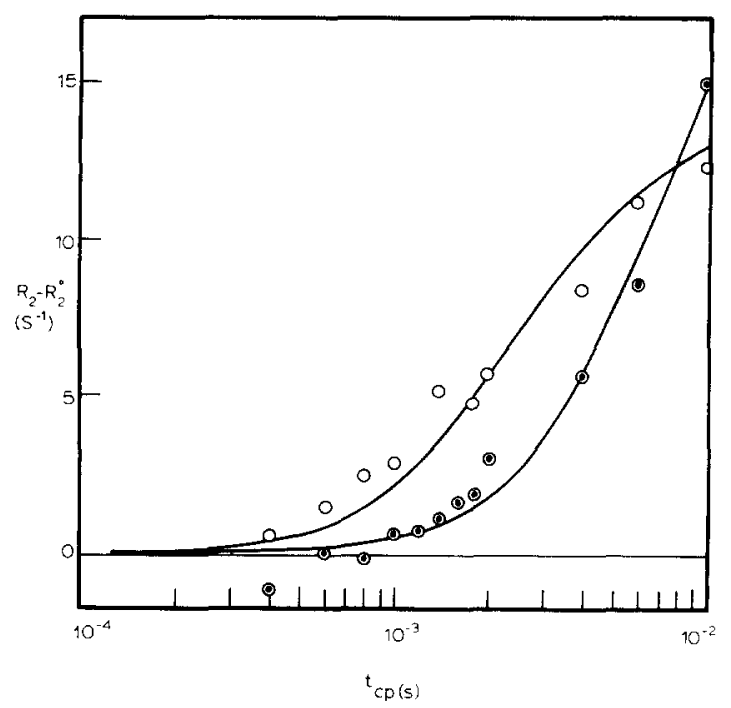

Fig. 8. Results of type II experiments for the chloroplast suspension described in the legend of Fig. 7 . The sample contains $250 \mu \mathrm{l}$ chloroplast suspension plus $20 \mu \mathrm{l}$ shift reagent. $T=25^{\circ} \mathrm{C}$, proton frequency equals 60.0 MHz. The solid line is a computer fit using Eqn. 4 and the theoretical parameters listed in Table 1 .

$\tau_{\mathrm{B}},\left(\delta^{2} V_{\mathrm{i}}\right), V_{\mathrm{m}}$, and $R_{2}^{0}$. Rapid convergence of the iteration procedure requires a reasonably accurate choice of initial parameters. This was accomplished using a computer graphics terminal to generate plot webs corresponding to a systematic variation of the parameters. In addition to providing good starting values, the plot webs served to ensure that the least-squares program was not iterating to a local minimum.

The error analysis showed that $V_{\mathrm{i}}$ and $V_{\mathrm{m}}$ are strongly covariant and that volumes computed from the least-squares procedure have little quantitative significance if iterations are carried out simultaneously on both parameters.

TABLE I

SUMMARY OF INTERNAL VOLUMES, OXYGEN ACTIVITY AND MEAN RESIDENCE TIMES OF WATER MOLECULES IN NATIVE AND NH $\mathrm{N}_{2} \mathrm{OH}-E X T R A C T E D$ CHLOROPLASTS

\begin{tabular}{|c|c|c|c|c|c|}
\hline \multirow[t]{2}{*}{ Preparation } & \multicolumn{2}{|c|}{$\begin{array}{l}\text { Activity (mol } \mathrm{O}_{2} \\
\left.\mathrm{mg}^{-1} \mathrm{Chl} \cdot \mathrm{h}\right)\end{array}$} & \multirow[t]{2}{*}{$T\left({ }^{\circ} \mathrm{C}\right)$} & \multirow[t]{2}{*}{$\tau_{\mathrm{B}}(\mathrm{ms})$} & \multirow[t]{2}{*}{$V_{\mathbf{i}}\left(\mu \mathrm{l} \cdot \mathrm{mg}^{-1} \mathrm{Chl}\right)$} \\
\hline & Coupled & Uncoupled & & & \\
\hline \multirow[t]{2}{*}{$\begin{array}{l}\mathrm{NH}_{2} \mathrm{OH} / \mathrm{EDTA} \\
\left(2.190 \mathrm{mg} \mathrm{Chl} \cdot \mathrm{ml}^{-1}\right)\end{array}$} & 0 & 0 & $25^{\circ} \mathrm{C}$ & $\begin{array}{l}1.17 \pm 0.08 * \\
1.10 \pm 0.08 * *\end{array}$ & $\begin{array}{l}160 * \\
114 * *\end{array}$ \\
\hline & & & $3^{\circ} \mathrm{C}$ & $\begin{array}{l}2.85 \pm 0.4 * \\
2.67 \pm 0.4 * *\end{array}$ & $\begin{array}{r}106 * \\
73 * *\end{array}$ \\
\hline \multirow[t]{2}{*}{$\begin{array}{l}\text { Native chloroplasts } \\
\left(2.500 \mathrm{mg} \mathrm{Chl} \cdot \mathrm{ml}^{-1}\right)\end{array}$} & 95 & 361 & $25^{\circ} \mathrm{C}$ & $\begin{array}{l}1.16 \pm 0.3 * \\
1.04 \pm 0.3 * *\end{array}$ & $\begin{array}{r}148 * \\
90 * *\end{array}$ \\
\hline & & & $3^{\circ} \mathrm{C}$ & $\begin{array}{l}1.79 \pm 0.4 * \\
1.70 \pm 0.4 * *\end{array}$ & $\begin{array}{r}114^{*} \\
76^{* *}\end{array}$ \\
\hline
\end{tabular}

* Calculated assuming $V_{\mathrm{m}}=100 \mu \mathrm{l} \cdot \mathrm{mg}^{-1} \mathrm{Chl}$.

$* * V_{\mathrm{m}}=150 \mu \mathrm{l} \cdot \mathrm{mg}^{-1} \mathrm{Chl}$. 
For this reason, $V_{\mathrm{m}}$ was subsequently fixed at one of two values, $100 \mu \mathrm{l} \cdot \mathrm{mg}^{-1}$ Chl or $150 \mu \mathrm{l} \cdot \mathrm{mg}^{-1} \mathrm{Chl}$, and iterations were carried out on the parameters $\tau_{\mathrm{B}}, V_{\mathrm{i}}$ and $R_{2}^{0}$. Fitted values of $\tau_{\mathrm{B}}$ and $V_{\mathrm{i}}$, corresponding to both assumed values of $V_{\mathrm{m}}$ are given in Table I along with standard deviations from the variancecovariance matrix.

From an inspection of Table I it is evident that the absolute value of $V_{\mathrm{i}}$ has a strong inverse dependence on the assumed value of $V_{m}$, reflecting the covariance of these parameters. Another limitation on the absolute accuracy of the calculated internal volume is that $V_{\mathrm{i}}$ appears as the product $\left(\delta^{2} V_{\mathrm{i}}\right)$ in the fast-exchange relations. Thus the estimation of $V_{\mathrm{i}}$ requires an accurate knowledge of the transmembranal shift. $\delta$ was measured from the concentration dependence of the proton shift, relative to the resonance of benzene in a sealed capillary, induced in the buffer by $\operatorname{Dy}(\mathrm{en})^{3+}$. This value could be inappropriate to chloroplast suspensions if $\mathrm{Dy}(\mathrm{en})^{3+}$ binds to the chloroplast membrane. Such binding seems likely since $\mathrm{Mn}^{2+}$ has been reported to bind weakly to sites on the external thylakoid membrane [35], and trivalent metal ions exert a profound effect on grana stacking [36]. Thus the absolute values calculated for internal volumes probably have little quantitative significance.

On the other hand, $\tau_{\mathrm{B}}$ is determined essentially independently of both $\delta$ and $V_{\mathrm{m}}$ from the inflection point of the $R_{2}-R_{2}^{0}$ vs. $t_{\mathrm{cp}}$ curve. The insensitivity of $\tau_{\mathrm{B}}$ to large variations in $V_{\mathrm{m}}$ is borne out by the results in Table I. Thus the standard deviations obtained from the fitting procedure are considered to be a realistic measure of the uncertainty in $\tau_{\mathrm{B}}$.

It should be stressed that all of the theoretical parameters, $\tau_{\mathrm{B}},\left(\delta^{2} V_{\mathrm{i}}\right)$, and $R_{2}^{0}$, are determined reasonably accurately by the data of the $t_{\mathrm{cp}}$-variation experiment alone. Thus a simultaneous fit of the parameters to the data of the two independent experiments provides a stringent test of self-consistency of the overall analysis.

\section{Temperature variation in the Carr-Purcell experiment}

The predicted effect of temperature variation on the Carr-Purcell experiment is evident from the theoretical plots in Fig. 3. At lower temperature, the inflection point of the $R_{2}-R_{2}^{0}$ vs $t_{\mathrm{cp}}$ plot is shifted to longer $t_{\mathrm{cp}}$ values, and the value of $R_{2}-R_{2}^{0}$ in the limit of long $t_{\mathrm{cp}}$ is increased. Thus the curves intersect, at least if $\delta^{2} V_{\mathrm{i}}$ is independent of temperature. The data confirm this qualitative prediction. As expected, the fitted value of $\tau_{\mathbf{B}}$ lengthens by a factor of, roughly, two between $25^{\circ} \mathrm{C}$ and $3^{\circ} \mathrm{C}$.

Fitted values of the internal volume are not independent of temperature, but are systematically smaller, by a factor of about $30 \%$, at the lower temperature. It has been pointed out above that absolute values of $V_{i}$ are of little quantitative significance because of uncertainties in the magnitudes of $\delta$ and $V_{\mathrm{m}}$. Relative values of $V_{\mathrm{i}}$ are likely to be much more reliable however, since both $\delta$ and $V_{\mathrm{m}}$ are probably nearly temperature-independent (the near temperatureindependence of measured values of $\delta$ has been described above). For this reason we believe that the observed decrease in $V_{i}$ as temperature is lowered is significant and indicates a temperature-dependent shrinkage of the loculus.

\section{Suspensions of native chloroplasts}

Results from experiments utilizing unextracted chloroplasts that were active 
in oxygen evolution are shown in Figs. 7 and 8 and Table I. Generally, the data from dark-adapted native chloroplasts were quite similar to those from $\mathrm{NH}_{2} \mathrm{OH}$ / EDTA-treated chloroplasts, except that fluctuations of the order of $20-30 \%$ in both the linewidth and $R_{2}$ were frequently noted. These effects were accentuated by exposure to light but were difficult to suppress, even when samples were preincubated for $10-12 \mathrm{~min}$ at $25^{\circ} \mathrm{C}$ in the dark and carefully shielded from light during measurements.

Fig. 7 shows an example of the kind of fluctuation encountered. The jump at $V_{\mathrm{SR}}=50 \mu \mathrm{l}$ presumably reflects changes, either in the internal volume or in the residence time in the internal phase, associated with low levels of electron transport. Except for the difference in stability, no significant difference in $\tau_{\mathrm{B}}$ or $V_{\mathrm{i}}$ was observed between active and $\mathrm{NH}_{2} \mathrm{OH} /$ EDTA-treated chloroplasts.

\section{Discussion}

Our values of the internal thylakoid volume are significantly larger than those measured by Gaensslen and McCarty [37,38], who found values of $35 \mu \mathrm{l} / \mathrm{mg} \mathrm{Chl}$ and $80 \mu \mathrm{l} / \mathrm{mg} \mathrm{Chl}$ for dark-and light-adapted chloroplasts respectively by measuring the amount of ${ }^{3} \mathrm{H}$-labelled water that sediments with the thylakoids. As pointed out above, the volumes derived from the NMR measurements are considered tentative because of uncertainties in the membrane volume and the transmembranal shift. The short measured value of $\tau_{\mathrm{B}}$ in the NMR experiments similarly suggests a possible source of serious uncertainty in the experiments of Gaensslen and McCarty. In their experiments, thylakoids containing tritiated water were isolated by centrifugation through a silicone layer. Since the silicone is osmotically inactive, extensive dehydration may have occurred during this step. Thylakoids remain in the silicone layer for times of the order of seconds, while the mean lifetime of water molecules in the inner thylakoid space prior to escape is the order of milliseconds. Thus dehydration seems probable. It is also surprising that a larger internal volume was found for light-adapted than for dark-adapted thylakoids, since electron-microscopic studies indicate that the volume of the loculus shrinks upon illumination [38]. The development of other independent techniques for measuring the internal volume would be useful to clarify this question.

\section{Effects of magnetic susceptibility on the Carr-Purcell experiment}

A second mechanism unrelated to chemical exchange that can in general lead to a $t_{\mathrm{cp}}$ dependence of $R_{2}$ is the diffusion of water protons through magnetic field gradients arising from magnetic susceptibility changes at phase boundaries of the thylakoid stacks. A nucleus diffusing through rapidly varying field gradients experiences periodic fluctuations in its Larmor frequency that are physically similar to the chemical shift variations experienced when the nucleus undergoes chemical exchange. Such effects have been observed in muscle [39] and in tubes of packed glass beads [40].

As a control to determine the magnitude of magnetic susceptibility effects in chloroplast suspensions, $R_{2}$ as a function of $t_{\mathrm{cp}}$ was measured in a sample that lacked shift reagent but contained an equal concentration of $\mathrm{La}^{3+}$. The diamagnetic susceptibility of $\mathrm{La}^{3+}$ (which is nonparamagnetic) is essentially the 
same as that of $\mathrm{Dy}^{3+}$. This experiment showed that susceptibility effects do not contribute appreciably to the $t_{\mathrm{cp}}$ dependence of $R_{2}$ in thylakoid suspensions.

Whether magnetic susceptibility can promote a $t_{\mathrm{cp}}$-dependence of $R_{2}$ depends on the characteristic particle dimensions of the heterogeneous system. Thylakoid stacks are two to three orders of magnitude smaller than the glass beads studied by Glasel and Lee [40] and fluctuation of the precession frequency due to diffusion is much more rapid than in that system (the timescale of fluctuations is roughly $5 \mu \mathrm{s}$ vs. $50 \mathrm{~ms}$ ). Since the inflection point in a $t_{\mathrm{cp}}$ experiment occurs when $t_{\mathrm{cp}} \cong 2 \tau$, this difference causes the susceptibility contribution to $R_{2}$ to be virtually independent of $t_{\mathrm{cp}}$. It is possible that a $t_{\mathrm{cp}}$ independent contribution may be present in both $R_{2}^{0}$ and in the linewidth, however.

\section{Conclusion}

The $\operatorname{Dy}(\mathrm{en})^{3+}$ shift reagent provides a useful method for studying transmembranal water exchange in thylakoid suspensions. This ion is impermeant toward the thylakoid membrane, it is inert as a redox reagent in physiologic media, and it produces large chemical exchange effects in NMR linewidths and $T_{2}$ values at millimolar concentrations. The methods reported here are considerably more versatile and accurate for measuring transmembranal water exchange than previous methods,..particularly when exchange lifetimes are in the millisecond or submillisecond range. This timescale is characteristic of subcellular particles. The advantage in rapid exchange situations results primarily from the extremely large molar shift of Dy(II). The technique is equally applicable to particles possessing a complex ultrastructure as to spherical vesicles.

Measurements of the mean lifetime of water molecules in the inner thylakoid space have given values near $1.1 \mathrm{~ms}$ at $25^{\circ} \mathrm{C}$ with an activation energy of about $5 \mathrm{kcal} / \mathrm{mol}$. Measurements of the relative internal volume of dark-adapted thylakoids indicate that this volume is not temperature-independent, but shrinks by about $30 \%$ as the temperature drops from $25^{\circ} \mathrm{C}$ to $3^{\circ} \mathrm{C}$.

\section{Acknowledgement}

This research was supported in part by a grant from the National Science Foundation (PCM78-7909).

\section{References}

1 Wydrzynski, T., Zumbulyadis, N., Schmidt, P.G. and Govindjee (1975) Biochim. Biophys. Acta 408, 349-354

2 Wydrzynski, T., Zumbulyadis, N., Schmidt, P.G., Gutowsky, H.S. and Govindjee (1976) Proc. Natl. Acad. Sci. U.S.A. 73, 1196-1198

3 wydrzynski, T., Govindjee, Zumbulyadis, N., Schmidt, P.G. and Gutowsky, H.S. (1976) Am, Chem. Soc. Symp. Ser, 34, 471-482

4 Wydrzynski, T.J., Marks, S.B., Schmidt, P.G., Govindjee and Gutowsky, H.S. (1978) Biochemistry 17 , 2155-2162

5 Conlon, T. and Outhred, R. (1972) Biochim. Biophys. Acta 288, 354-361

6 Shporer, M. and Civan, M.M. (1975) Biochim. Biophys. Acta 385, 81-87

7 Andrasko, J. (1976) Biochim. Biophys. Acta 428, 304-311 
8 Guikema, J.A. and Yocum, C.F. (1976) Biochemistry 15, 362-367

9 Horton, P. and Croze, E. (1977) Biochim. Biophys. Acta 462, 86-101

10 Vold, R.L., Vold, R.R. and Simon, H.E. (1973) J. Magn. Reson. 11, 283-298

11 Simpson, J.H. and Carr, H.Y. (1958) Phys. Rev. 111, 1201-1202

12 Meiboom, S. (1961) J. Chem. Phys. 34, 375-388

13 Reuben, J. and Fiat, D. (1967) J. Chem. Phys. 47, 5440-5441

14 Reuben, J. and Fiat, D. (1969) J. Chem. Phys. 51, 4909-4917

15 Dwek, R.A. (1973) Nuclear Magnetic Resonance in Biochemistry, Chapter 4, Oxford University Press, Oxford

16 Bleaney, B. (1972) J. Magn. Reson. 8, 91-100

17 Thomson, W.W. (1972) in Dynamic Aspects of Plant Ultrastructure (Robards, A.W., ed.), pp. 138177, Mc Graw-Hill, New York

18 Park, R.B. and Sane, P.V. (1971) Ann. Rev. Plant Physiol. 22, 395-430

19 Kreutz, W. (1970) Adv. Bot. Res. 3, 53-169

20 Paolillo, D.J., Jr, and Falk, R.H. (1966) Am. J. Bot. 53, 173-180

21 Paollilo, D.J., Jr., MacKay, N.C. and Graffius, J.R. (1969) Am. J. Bot. 56, 344-347

22 Paolillo, D.J., Jr., Falk, R.H. and Reighard, J.A. (1967) Trans. Am. Microse. Soc. 86, 225-232

23 Paolillo, D.J., Jr and Reighard, J.A. (1967) Can. J. Bot. 45, 773-782

24 Emsley, J.W., Feeney, J. and Sutcliff, L.H. (1965) High Resolution Nuclear Magnetic Resonance Spectroscopy, Chapter 9, Pergamon, New York

25 Anderson, P.W. (1954) J. Phys. Soc. Jap. 9, 316-324

26 Hoffman, R.A. (1970) Adv. Magn. Reson. 4, 87-200

27 Rogers, M.T. and Woodbrey, J.C. (1962) J. Phys. Chem. 66, 540-546

28 Meiboom, S. and Gill, D. (1958) Rev. Sci. Instrum, 29, 688-690

29 Farrar, T.C. and Becker, E.D. (1971) Pulse and Fourier Transform NMR, Academic Press, New York

30 Abragam, A. (1961) The Principles of Nuclear Magnetism, Oxford University Press, Oxford

31 Bloom, M., Reeves, L.W. and Wells, E.J. (1965) J. Chem. Phys. 42, 1615-1624

32 Allerhand, A. and Gutowsky, H.S. (1964) J. Chem. Phys. 41, 2115-2126

33 Allerhand, A. and Gutowsky, H.S. (1965) J. Chem. Phys. 42, 1587-1599

34 Gutowsky, H.S., Vold, R.L. and Wells, E.J. (1965) J. Chem. Phys. 43, 4107-4125

35 Blankenship, R.E., Babcock, G.T. and Sauer, K. (1975) Biochim. Biophys. Acta 387, 165-175

36 Barber, J. and Chow, W.S. (1979) FEBS Lett. 105, 5-10

37 Gaensslen, R.E. and McCarty, R.E. (1971) Arch. Biochem. Biophys. 147, 55-65

38 Murakami, S. and Packer, L. (1970) J. Cell Biol, 47, 332-351

39 Packer, K.J. (1973) J. Magn. Reson. 9, 438-443

40 Glasel, J.A. and Lee, K.H. (1975) J. Am. Chem. Soc. 96, 970-978 